\title{
Composição corporal por meio da absortometria radiológica de dupla energia em idosos institucionalizados do Hospital Santo Antônio
}

\author{
Body composition through dual energy radiological absorptiometry of institutionalized \\ elderly at Hospital Santo Antônio
}

Sara Moreira Anunciação ${ }^{1^{*}}$, Helton Estrela Ramos²

${ }^{1}$ Nutricionista. Mestranda do Programa de Pós-Graduação de Processos Interativos de Órgãos e Sistema. Instituto de Ciências da Saúde Universidade Federal da Bahia - UFBA; ${ }^{2}$ Doutor em Endocrinologia Clínica. Professor Associado do Instituto de Ciências da Saúde e do Programa de Pós-graduação em Processos Interativos dos Órgãos e Sistemas. Instituto de Ciências da Saúde. Universidade Federal da Bahia-UFBA

\begin{abstract}
Resumo
Introdução: o processo de envelhecimento tem sido constante objeto de estudos epidemiológicos, em função do recrudescimento do número de idosos na população, resultando numa incessante busca de instituições de longa permanência. $O$ uso de métodos de avaliação nutricional e de composição corporal em idosos dessas instituições pode ser considerado um diferencial na assistência multimodal. Objetivo: avaliar a composição corporal de idosos institucionalizados por meio da Absortometria Radiológica de Dupla Energia, atendidos do Hospital Santo Antônio, na cidade de Salvador, Bahia. Metodologia: estudo descritivo, analítico, corte transversal, com 13 idosos de ambos os sexos da referida instituição. Aplicou-se questionário sociodemográfico e de dados clínicos e os idosos foram submetidos a DXA, obedecendo a protocolos padrão para realização do exame. Resultados: predominância do sexo feminino $(61 \%)$ e idade média de $79,5(\mathrm{DP}=7,0)$. As mulheres se destacaram por excesso de gordura corporal $(61,5 \%)$ e, no que tange à reserva de massa muscular, a distribuição percentual e absoluta foi dividida de forma igual para ambos os sexos (50\%). Conclusões: foi possível observar que grande parte dos idosos avaliados, e em especial mulheres, apresentou excesso de tecido corporal gorduroso, considerando todas as variáveis relacionadas à massa gordurosa corporal reveladas pela DXA. Estudos dentro dessa temática, no último ciclo de vida mais vulnerável são de suma importância, uma vez que o número populacional de idosos tem assumido nos últimos anos um crescimento exponencial.

Palavras-chave: Composição corporal. Envelhecimento. Idosos. Saúde do idoso institucionalizado.
\end{abstract}

\begin{abstract}
Introduction: the aging process has been a constant object of epidemiological studies, due to the increase in the number of elderly people in the population, resulting in an incessant search for long-term care facilities. The use of methods for nutritional assessment of body composition in the elderly in these institutions can be considered a differential in multimodal care. Objective: assessing the body composition of elderly institutionalized people using dual energy X-ray absorptiometry at Santo Antônio Hospital, in the city of Salvador, Bahia. Methodology: descriptive, analytical, cross-sectional study with 13 elderly people male and female from that institution. A sociodemographic and clinical data questionnaire was applied and the elderly were submitted to DXA, following standard protocols for the examination. Results: prevalence of females (61\%) and mean age of $79.5(S D=7.0)$. Women stood out due to excess body fat (61.5\%) and, with regard to muscle mass reserve, the percentage and absolute distribution was equally divided for men and women (50\%). Conclusion: it was possible to observe that most of the elderly evaluated, and especially women, had excess body fat tissue, considering all variables related to body fat mass revealed by DXA. Studies into this theme are crucial in the last most vulnerable life cycle, since the number of elderly population has assumed an exponential growth in recent years.

Keywords: Body composition. Early. Elderly. Health of the institutionalized elderly.
\end{abstract}

\section{INTRODUÇÃO}

O processo de envelhecimento tem sido constante objeto de estudos epidemiológicos, em função do recrudescimento do número de idosos na população e devido ao surgimento de morbidades que acompanha o aumento dessa taxa de crescimento.

Conforme apontam os dados atuais, o número de pessoas acima de 60 anos poderá ultrapassar mundial-

Correspondente/Corresponding: *Sara Moreira Anunciação-Instituto de Ciências da Saúde - Universidade Federal da Bahia - End; Av. Reitor Miguel Calmon, s/n - Vale do Canela - 40.110-902 Salvador - BA - Tel: (71) 99959-0019-E-mail: sanunciacao@ufba.br mente de 901 milhões para 1,4 bilhão, com estimativa de atingir 2,1 bilhões em 2050 (LORENZO-LOPEZ et al., 2017). Melhorias concernentes à qualidade de vida podem ser um dos fatores desse crescimento global; em contrapartida aumenta a incidência de morbidades relacionadas ao processo de envelhecimento (VALENTINI et al., 2018).

O envelhecer, seja patológico, seja fisiológico, aumenta a propensão do desenvolvimento de alterações clínicas, sobretudo funcionais e nutricionais, cenário que requer cuidados especiais e individualizados (; DENIC; GLASSOCK; RULE, 2016; OLIVEIRA NETO et al., 2020).

Devido a essas modificações, a vigilância do estado nutricional do idoso é específica, assim como o tratamen- 
to. É nesse ciclo de vida que irrompem frequentes complicações de afecções crônicas e problemas nutricionais extremos, como a sarcopenia e a obesidade. A redução da ingestão alimentar de nutrientes envolvidos em elementos da composição tecidual (epitelial, muscular e óssea), inapetência, disfunções metabólicas, hormonais, mentais, digestórias e de composição corporal atuam de forma sinérgica e integrada, contribuindo para a fragilidade da saúde do idoso, num contexto geral (MUNDI et al., 2018).

$\mathrm{O}$ acelerado e crescente aumento de idosos na população, em consonância com a mudança da dinâmica da família brasileira, culminam, atualmente, em uma incessante busca de instituições de longa permanência para idosos (ILPI).

As ILPI surgiram como uma das formas de cuidado integral e sistêmico, prática reconhecida pela Sociedade Brasileira de Geriatria e Gerontologia (SBGG) e regulamentada pela Resolução da Diretoria Colegiada (RDC) n. 283, de 26 de setembro de 2005, da Agência Nacional de Vigilância Sanitária (ANVISA) (BRASIL. MINISTÉRIO DA SAÚDE, 2005; CAMARANO; KANSO, 2010).

A ILPI precisa dispor de uma equipe multiprofissional destinada à assistência integral, tendo em vista as peculiaridades da saúde do idoso e a possível potencialização dos efeitos acarretados pela mudança de ambiência e de hábitos na saúde do indivíduo. O complexo fenômeno de mudanças biopsicossociais do idoso, associado ao processo de institucionalização, configuram quadro de risco de degeneração do estado nutricional.

Neste sentido, no âmbito da abordagem nutricional, instrumentos de avaliação nutricional validados e sensíveis para essa faixa etária são cruciais visando ao correto diagnóstico nutricional, para que o plano terapêutico seja o mais individualizado possível e, consequentemente, eficaz (SILVA et al., 2019).

O envelhecimento aumenta a tendência de modificações de compartimentos corporais, tendo a redução da estatura, massa muscular total e massa gorda periférica como consequências típicas dessa faixa etária. Em contrapartida, ocorre aumento do tecido gorduroso omental, acarretando, no indivíduo, alta propensão de doenças crônicas (SOPEÑA et al., 2018).

Frente a isto, o monitoramento de indicadores nutricionais mais diretamente afetados com o envelhecimento, como avaliação do tecido muscular e de gordura total, é relevante na detecção e tratamento de enfermidades clássicas do envelhecimento, a exemplo da sarcopenia, obesidade sarcopênica, osteoporose, dentre outras.

Os índices e indicadores antropométricos, como por exemplo, índice de massa corporal (IMC), pregas cutâneas e medidas de circunferência, são amplamente utilizados em estudos populacionais, em função da fácil aplicabilidade, baixo custo e por dispensarem altas habilidades técnicas. No entanto, têm baixa sensibilidade e precisão, sobretudo quando se trata das alterações da distribuição de massa muscular e adiposa, cuja ocorrência em idosos é mais acentuada (KURIYAN, 2018).
Desse modo, para avaliação categórica da composição corporal, dois métodos mais indicados são a bioimpedância (BIA) e absortometria radiológica de dupla energia (DEXA ou DXA), sendo este determinado como padrão-ouro para a avaliação de composição corporal em nível molecular na população em geral. A DXA é considerada como método rápido, não invasivo, de baixa exposição à radiação, além de discernir massa gorda, massa magra e conteúdo mineral corporal (ZHOU; HOGLUND; CLYNE, 2019). Por outro lado, poucos estudos adotam a DXA para avaliação corporal, possivelmente, devido à dificuldade do acesso e custo moderado do equipamento.

O uso de métodos de avaliação nutricional de composição corporal em idosos institucionalizados pode ser considerado um diferencial na assistência multimodal, sobretudo quando se trata de indivíduos dependentes de cuidados delegados pela família ou pelo próprio idoso. As informações obtidas pela DXA oferecem subsídios para todos os campos profissionais, ou seja, auxiliam no norteamento da prescrição dietética individualizada, medicamentosa e atividade física, podendo, por sua vez, resultar em terapêuticas mais assertivas e eficazes.

Sendo assim, o objetivo deste estudo foi avaliar a composição corporal de idosos por meio de um método direto de avaliação da composição corporal, DXA, residentes em instituição de longa permanência do Hospital Santo Antônio, da cidade do Salvador, Bahia.

\section{METODOLOGIA}

\section{Amostra e coleta de dados}

Trata-se de estudo descritivo, analítico, corte transversal, conduzido em janeiro de 2021, com o recrutamento de idosos de ambos os sexos. A amostra foi composta por 13 idosos residentes em instituição de longa permanência do Hospital Santo Antônio das Obras Sociais Irmã Dulce, cidade do Salvador, Bahia, Brasil.

A instituição de longa permanência do Hospital Santo Antônio das Obras Sociais Irmã Dulce faz parte de uma subunidade da organização, com assistência médica integral e multidisciplinar, sendo caracterizada como instituição filantrópica, e que atualmente acolhe 52 idosos. Este estudo foi executado de acordo com a Resolução CNS 466/12 da Comissão Nacional de Ética e Pesquisa (CONEP), sendo também aprovado pelo Comitê de Ética em Pesquisa (CEP), do Instituto de Ciências da Saúde, UFBA, cujo número do Certificado de Apresentação de Apreciação Ética (CAAE) é 93016218.9.0000.5662.

\section{Critérios de inclusão e exclusão}

Os critérios de inclusão dos idosos neste estudo foram: idade superior a 60 anos, residentes na ILPI em questão e que tenham concordado em participar do estudo, com o termo de consentimento livre e esclarecido (TCLE) devidamente assinado. Para os critérios de exclu- 
são, foram considerados os seguintes aspectos: cuidados paliativos ou fim de vida; doença renal crônica dialítica ou qualquer morbidade associada à retenção hídrica; acamados; que não seguissem protocolo para realização da DXA; sem o TCLE devidamente preenchido; peso corporal e altura acima da capacidade da DXA; que tenham feito uso de contraste ou sido submetidos a exames de imagem com contraste.

\section{Dados sociodemográficos e clínicos}

Os participantes responderam a um questionário validado e estruturado, onde constavam informações sobre idade, sexo, comorbidades, uso de medicamentos, exames clínicos e estilo de vida.

\section{Avaliação da composição corporal}

Utilizou-se a absortometria de raios-X de dupla energia (DXA). Os pacientes foram encaminhados a uma clínica particular de imagem da cidade do Salvador, sendo acompanhados pelo profissional da enfermagem da instituição de procedência. Na clínica, os exames foram realizados por técnicos treinados e que dominavam o manejo do equipamento calibrado, conforme recomendação do fabricante.

O exame foi realizado pela técnica de absorção de dois feixes de baixa energia emitidos por raios- $X$, em densitômetro modelo Podigy Advance Lunar-GE. Utilizou-se protocolo de corpo inteiro por cerca de 10 minutos, onde os idosos foram colocados em posição supina, com os braços estendidos perto do tronco e mais baixo, com ligeira abdução dos pés.

Não foram submetidos ao exame idosos com peso superior a $136 \mathrm{~kg}$ ou altura de $196 \mathrm{~cm}$, bem como aqueles que haviam feito exames com contraste ou de medicina nuclear no período anterior 72 horas. Além disso, os idosos participantes foram orientados a não realizar nenhum tipo de atividade física anterior ao exame, assim como a dirigir-se à clínica de preferência em jejum.

Adornos metálicos foram retirados e todos os participantes utilizaram vestimentas fornecidas pela clínica (avental). Solicitou-se ao idoso esvaziamento da bexiga urinária antes do procedimento.

Para avaliar o índice de massa gorda corporal em kg/ $\mathrm{m}^{2}$ foi utilizado Fat Mass Index (FMI). Utilizou-se o estudo de Kelly et al. (2009) como referência para FMI, com os seguintes pontos de corte: deficit severo de gordura, homens inferior a $2,0 \mathrm{~kg} / \mathrm{m}^{2}$ e mulheres inferior $3,5 \mathrm{~kg} / \mathrm{m}^{2}$; deficit moderado de gordura, homens de $2,0 \mathrm{~kg} / \mathrm{m}^{2}$ a 2,3 $\mathrm{kg} / \mathrm{m}^{2}$ e mulheres de $3,5 \mathrm{~kg} / \mathrm{m}^{2}$ a $4,0 \mathrm{~kg} / \mathrm{m}^{2}$; déficit leve de gordura, homens de $2,3 \mathrm{~kg} / \mathrm{m}^{2}$ a $3,0 \mathrm{~kg} / \mathrm{m}^{2}$ e mulheres de $4,0 \mathrm{~kg} / \mathrm{m}^{2}$ a $5,0 \mathrm{~kg} / \mathrm{m}^{2}$; normal, homens de $3,0 \mathrm{~kg} / \mathrm{m}^{2}$ a 6,0 $\mathrm{kg} / \mathrm{m}^{2}$ e mulheres de $5,0 \mathrm{~kg} / \mathrm{m}^{2}$ a $9,0 \mathrm{~kg} / \mathrm{m}^{2}$; sobrepeso, homens de $6,0 \mathrm{~kg} / \mathrm{m}^{2}$ a $9,0 \mathrm{~kg} / \mathrm{m}^{2}$ e mulheres de $9,0 \mathrm{~kg} /$ $\mathrm{m}^{2}$ a $13,0 \mathrm{~kg} / \mathrm{m}^{2}$; obesidade classe 1 , homens de $9,0 \mathrm{~kg} /$ $\mathrm{m}^{2}$ a $12,0 \mathrm{~kg} / \mathrm{m}^{2}$ e mulheres de $13,0 \mathrm{~kg} / \mathrm{m}^{2}$ a $17,0 \mathrm{~kg} / \mathrm{m}^{2}$; obesidade classe 2 , homens de $12,0 \mathrm{~kg} / \mathrm{m}^{2}$ a $15,0 \mathrm{~kg} / \mathrm{m}^{2}$ e mulheres de $17,0 \mathrm{~kg} / \mathrm{m}^{2}$ a $21,0 \mathrm{~kg} / \mathrm{m}^{2}$; obesidade classe 3 , homens superior a $15,0 \mathrm{~kg} / \mathrm{m}^{2}$ e mulheres superior a $21 \mathrm{~kg} / \mathrm{m}^{2}$.

O índice de massa magra é traduzido pelo índice de Baumgartner (IB) que considera a massa magra apendicular (ALM) representada pela massa magra do braço bilateral e perna bilateral e se baseia pelo preconizado por Cruz-Jentof et al. (2010): maior que $5,5 \mathrm{~kg} / \mathrm{m}^{2}$ para mulheres não sugestivo de deficit de massa muscular e para homens, maior que $7,26 \mathrm{~kg} / \mathrm{m}^{2}$.

\section{Análise estatística}

Os dados coletados foram tabulados e analisados no Microsoft Excel 2016.

Utilizou-se estatística descritiva, sendo obtidos valores de média, mediana, desvio padrão amostral (DP) e coeficiente de variação (CV). Não foi aplicada inferência estatística, tendo em vista a amostra não probabilística, cuja escolha dos elementos da amostra não foi feita de forma arbitrária e, sim, de acordo com a conveniência do pesquisador.

\section{RESULTADOS}

\section{Características da amostra}

Um total de 13 idosos residentes na instituição foram incluídos neste estudo, sendo a maioria do sexo feminino (61\%) e idade média de 79,5 (DP=7,0). Com relação à presença de doenças crônicas, 54\% (7) tinham diagnóstico clínico de hipertensão arterial sistêmica (HAS), 38,5\% (5) diabetes mellitus tipo 2 (DM), 15,4\% (2) dislipidemia (DLP), 23\% (3) acidente vascular cerebral (AVC) e 7,7 \% (1) insuficiência renal crônica não-dialítica (IRC). Dessa forma, a HAS, DM e AVC, doenças crônicas e metabólicas, foram as mais prevalentes. A Tabela 1 , a seguir, descreve as variáveis sociodemográficas e clínicas, com respectivas médias, desvios-padrões e medianas.

Tabela 1 - Descrição da amostra de acordo com variáveis sociodemográficas e clínicas dos idosos da instituição de longa permanência do Hospital Santo Antônio, Salvador, 2021.

\begin{tabular}{|c|c|c|c|}
\hline Variáveis & $\begin{array}{c}n \\
(\%)\end{array}$ & $\begin{array}{c}\text { Média } \\
\text { (DP) }\end{array}$ & Mediana \\
\hline \multicolumn{4}{|l|}{ Sexo } \\
\hline Feminino & $8(61)$ & & \\
\hline Masculino & $5(39)$ & & \\
\hline Idade & & $79,5(7,0)$ & 80 \\
\hline \multicolumn{4}{|l|}{$\begin{array}{l}\text { Presença de } \\
\text { morbidades }\end{array}$} \\
\hline HAS & 7 (54) & & \\
\hline DM & $5(38,5)$ & & \\
\hline AVC & $3(23)$ & & \\
\hline DLP & $2(15,4)$ & & \\
\hline IRC & $1(7,7)$ & & \\
\hline
\end{tabular}

Fonte: Dados da pesquisa. 


\section{Composição corporal pela DXA}

Com relação aos dados de composição corporal obtidos pela DXA, os participantes apresentaram IMC médio de 27,0 (DP=6,6; CV= 24\%). O percentual de GCT foi de $31,7 \%$ ( $D P=10,3 ; C V=33 \%)$, FMI médio de 8,9 ( $D P=4,7$ $C V=53 \%)$, ALM médio 16,8 ( $D P=4,4 ; C V=26 \%$ ) e IB médio de 6,8 ( $D P=1,0 ; C V=15 \%)$.

Mediante a análise dos resultados e dos índices fornecidos pelos laudos da DXA dos idosos submetidos ao exame, observou-se que a maioria dos participantes, sobretudo mulheres, apresentou excesso de gordura corporal, ao se considerar no mínimo o sobrepeso.

Na Tabela 2, pode-se observar os valores médios com desvio padrão, coeficiente de variação e mediana de composição corporal, pela técnica de absorção de dois feixes de baixa energia emitidos por raios-X em densitômetro.

Tabela 2 - Dados, índices e indicadores de composição corporal dos idosos extraídos dos laudos da DXA.

\begin{tabular}{lrrc} 
Variáveis & Média (DP) & Mediana & CV (\%) \\
\hline Altura (cm) & $156(11,6)$ & 156 & 7 \\
Masculino & $166,2(9,6)$ & 165 & 6 \\
Feminino & $149,5(7,5)$ & 148,5 & 5 \\
Peso (kg) & $65,2(14,1)$ & 64,5 & 22 \\
Masculino & $69,1(13,1)$ & 68,5 & 19 \\
Feminino & $62,7(14,9)$ & 61,4 & 24 \\
IMC (Kg/m $\left.{ }^{2}\right)$ & $27,0(6,6)$ & 26,5 & 24 \\
Masculino & $24,9(3,3)$ & 26,1 & 13 \\
Feminino & $28,4(7,9)$ & 28 & 28 \\
Composição corporal & & & \\
GCT (\%) & $31,7(10,3)$ & 30,5 & 33 \\
Masculino & $27,1(7,4)$ & 29,3 & 27 \\
Feminino & $34,5(11,3)$ & 36,4 & 33 \\
FMI (kg/m $\left.{ }^{2}\right)$ & $8,9(4,7)$ & 8 & 53 \\
Masculino & $6,9(2,3)$ & 7,6 & 34 \\
Feminino & $10,1(5,5)$ & 9,4 & 54 \\
ALM (kg) & $16,8(4,4)$ & 15,7 & 26 \\
Masculino & $20,9(4,2)$ & 19,7 & 20 \\
Feminino & $14,1(1,9)$ & 14,2 & 13 \\
IB (kg/m $\left.{ }^{2}\right)$ & $6,8(1,0)$ & 6,6 & 15 \\
Masculino & $7,5(0,7)$ & 7,2 & 10 \\
Feminino & $6,4(1,0)$ & 6 & 15 \\
\hline
\end{tabular}

Fonte: Dados da pesquisa

A classificação da DXA por meio do FMI revelou: sobrepeso 1 mulher e 4 homens; obeso classe 1 apenas mulheres (2); obesos classe 2 somente 1 mulher e nenhum obeso classe 3 .

Ainda com relação à composição de gordura corporal pelo $\mathrm{FMI}, 61,5 \%$ (8) dos idosos apresentaram excesso no mínimo com o sobrepeso revelado pela DXA.

No que tange à reserva de massa muscular, a distribuição percentual e absoluta foi dividida de forma igual para ambos os sexos (50\%). Somente $30,8 \%$ dos idosos avaliados apresentaram IB sugestivo de deficit de massa muscular (dois idosos do sexo masculino e dois feminino).

\section{DISCUSSÃO}

Conhecer o estado nutricional de forma compartimentalizada possibilita a aplicação de relevantes condutas clínicas e nutricionais no cuidado à saúde, especialmente, no idoso, haja vista as modificações corporais influenciadas pelo processo de envelhecimento.

O uso da antropometria, como o habitual IMC, pode auxiliar no norteamento ou sinalização da necessidade de investigação mais aprofundada do estado nutricional do idoso, tendo em vista alteração da composição corporal inerente associada à idade. No entanto, o IMC apresenta limitações mais acentuadas nesse ciclo de vida, devido ao fenômeno natural de redistribuição da gordura corporal e da tendência à redução da massa magra, não sendo tão sensível para tal (SOPEÑA et al., 2018).

Diversos especialistas sobre o assunto defendem que a distinção dos segmentos corporais no idoso se torna mais interessante que o IMC na determinação do risco à saúde, principalmente em se tratando de morbidades crônicas (XU et al., 2020).

Os principais achados do estudo caracterizaram idosos com IMC médio de $27 \mathrm{~kg} / \mathrm{m}^{2}$ com DP=6,6 e CV=24\%, revelando um IMC sugestivo de indivíduos com excesso de peso, ao se ter como base as referências de Lipschitz (1994). Esta referência classifica idosos eutróficos com IMC de 22 a $27 \mathrm{~kg} / \mathrm{m}^{2}$ O IMC elevado em idosos tem sido constantemente correlacionado ao excesso de tecido adiposo e sua potencial interação como fator de risco cardiovascular (BONNEFOY; GILBERT, 2015).

Em vista disso, o presente estudo corroborou a prevalência de idosos com excesso de gordura corporal (61,5\%), sendo o FMI médio de $8,9 \mathrm{~kg} / \mathrm{m}^{2}$ com $\mathrm{DP}=4,7$ superando a marca de FMI de $6,0 \mathrm{~kg} / \mathrm{m}^{2}$, que categoriza homens com sobrepeso, e próximo ao limite de normalidade do ponto de corte $9,0 \mathrm{~kg} / \mathrm{m}^{2}$ para mulheres.

Comumente, identifica-se maior percentual de gordura corporal em mulheres idosas, ao invés dos homens, isto devido à diferença de hormônios estrogênicos, que com o envelhecimento diminuem e retardam o metabolismo lipídico (BARBOSA et al., 2015). Ainda com relação a Barbosa, no ano de 2015, observou-se excesso de gordura corporal nas idosas institucionalizadas em sua pesquisa.

Estudo utilizando dados antropométricos de idosos para avaliação da composição corporal observou que a distribuição do percentual de gordura corporal total apresentou-se maior no sexo feminino (38,18; $D P=4,498)$, em comparação ao sexo masculino $(27,98$; $D P=3,585)$ (SILVA et al., 2015).

Sopeña et al. (2018) analisaram a composição corporal por meio da BIA em mais de 100 idosos da rede de atenção primária e constataram que as mulheres idosas também superaram a média do percentual de gordura corporal: valores de 40,9\% (DP=0,9) em contraste com 31,9\% (DP=1,8) nos homens.

Em investigação mais recente, publicada por Silveira e outros pesquisadores em 2021, a qual utilizou dobras cutâ- 
neas, equações antropométricas preditivas, tomando como referência de método padrão ouro a $D X A$, revelou achados similares de percentual de corporal médio, $43,4 \%(D P=7,9)$ nas idosas contra $30,2 \%(D P=8,6)$ nos homens.

Ainda, nessa perspectiva, os achados de outro grupo de pesquisa em estudo realizado em 202 idosos, as mulheres avaliadas pela DXA tiveram um percentual de massa de gordura corporal significativamente maior do que os homens $(36,06 ; \mathrm{DP}=5,07$ versus 26,$24 ; \mathrm{DP}=5,91)$ (FANTIN et al., 2020).

A presente investigação dispõe de dados consonantes com os autores supracitados, cujo percentual de gordura corporal pela DXA foi de $34,5 \%(D P=11,3)$ em mulheres e $27,1 \%(D P=7,4)$ em homens.

Em paralelo ao percentual de gordura corporal, sua localização também define fatores de risco de saúde cardiometabólico, uma vez que há diferenças da distribuição em região androide e ginoide (LU et al., 2019). A DXA dos idosos do presente estudo revelou que $85 \%$ (11) apresentaram predomínio de gordura do tipo ginoide e $15 \%$ (2) androide, apontando elevado risco de síndrome metabólica e de resistência insulínica, em função da gordura troncular relacionada a visceral (LU et al., 2019).

Atualmente, a avaliação da distribuição da gordura corporal pela DXA é considerada de maior representatividade para eventos cardiovasculares, sendo a androide mais fortemente associada ao desequilíbrio de lipoproteínas séricas e aumento de triglicerídeos, quando comparada com a ginoide. Ademais, alguns estudo aventam a proteção da gordura ginoide contra doenças cardiovasculares (MIN; MIN, 2015).

Estes resultados são importantes para o estado de saúde do idoso institucionalizado, uma vez que pode predizer o aparecimento de novas doenças metabólicas e cardiovasculares, em especial nas mulheres, cujas alterações hormonais impõem propensão ao acúmulo de gordura em detrimento da massa muscular.

De forma consolidada na literatura, sabe-se que o excesso de gordura corporal tem forte associação com doenças cardiometabólicas. As adiponectinas e leptinas produzidas pelo tecido adiposo geralmente encontram-se em concentrações elevadas nos obesos. Esses hormônios apresentam íntima relação com risco cardiovascular e seus níveis mudam conforme evolui o processo de envelhecimento (FANTIN et al., 2020).

A diabetes mellitus foi a segunda doença mais frequente na amostra, cuja prevalência tem progredido em ritmo acelerado no mundo e sua forte associação com o envelhecimento tem sido estudada de forma recorrente (GUZIK; COSENTINO, 2018). As transformações de composição corporal inatas do envelhecimento surgem como mais um fator causal não modificável da diabetes, mas também como potencializador dos dados alarmantes epidemiológicos da doença. Destaca-se, inclusive, que o aumento de tecido adiposo no lugar da massa muscular no idoso pode ser não só fator determinante de risco, mas consequência da afecção (AL-SOFIANI et al., 2019).
Todos os idosos do estudo apresentaram alguma doença cardiovascular e metabólica, sendo a HAS, DM e AVC as mais prevalentes. Este achado, somado à alta prevalência de gordura corporal, pode traduzir o risco da população a novos eventos cardiovasculares ou, até mesmo, agravo do estado clínico e nutricional. O cenário endossa a relevância de um plano terapêutico multimodal e individualizado, uma vez que o grau de influência das alterações corporais e sua associação com doenças crônicas pode ser intensificado, a depender do sexo, idade e patologias subjacentes.

A distribuição da gordura corporal adentra outro assunto bastante comum nessa fase da vida: a queda da própria altura em idosos, associada não só a sua capacidade funcional, mas também ao seu estado de fragilidade corporal.

Recentemente foi publicado artigo, cujo escopo foi avaliar obesidade androide e ginoide e a incidência de quedas em idosas. Para os autores, a obesidade androide é um fator de risco para queda. Além disso, identificar a concentração da posição da gordura corporal faz diferença na proteção contra quedas em idosos (ginoide) (NERI et al., 2020). Desse modo, os idosos do estudo em questão têm alto risco de fraturas, escoriações e até mesmo de problemas mais complexos, como de imobilidade e de ordem psicológica. Isto demonstra quão importante a avaliação nutricional, esmiuçada pela DXA, pode subsidiar com informações de caráter preventivo, atravessando as questões metabólicas e correlacionando a composição corporal com variáveis funcionais e psicológicas, ou seja, de interesse de toda equipe multiprofissional.

O outro extremo ao excesso de gordura corporal em idosos seria a deficiência de massa muscular, que, inicialmente, era definida de forma reducionista como sarcopenia do idoso. Contudo, a definição de sarcopenia adquiriu, com o advento evolutivo das pesquisas científicas, conceitos mais amplos, tendo inclusive estratificação da gravidade e critérios para diagnóstico (NASIMI; DABBAGHMANESH; SOHRABI, 2019).

Dentro desse contexto, tem sido estudada amplamente a obesidade sarcopênica em idosos, cuja etiopatogenia está conjugada a aspectos relacionados à idade, como: mudanças na composição corporal, alterações estrogênicas e androgênicas, vias inflamatórias em hiperatividade e mecanismos moleculares deficientes de defesa contra o envelhecimento celular (BATSIS; VILLAREAL, 2018).

Na DXA, a ALM é utilizada para classificação da massa muscular, sendo o Índice de Baumgartner (IB) o indicador responsável pela classificação. O IB é obtido pela razão ALM/Altura. Baumgartner e seu grupo de pesquisadores desenvolveram em 1998 valores de corte de referência para o diagnóstico de sarcopenia na DXA (BAUMGARTNER et al., 1998).

Dentre os idosos avaliados, não foi identificada alta prevalência de deficiência de massa muscular, 30,8\% (4). A maior parte deles apresentou IB satisfatório para reserva de massa muscular, sendo a média de $7,5 \mathrm{~kg} / \mathrm{m}^{2}(\mathrm{DP}=0,7)$ 
em homens e $6,4 \mathrm{~kg} / \mathrm{m}^{2}(\mathrm{DP}=1,0)$ em mulheres. Decerto, o pequeno tamanho amostral, uma das limitações do estudo, impediu a observância do evento comum em idosos, a depleção de massa magra.

Ademais, a obesidade sarcopênica é mais prevalente em idosos com idade superior a 65 anos e nenhum dos idosos avaliados contava com idade inferior a 70 anos (média de idade de 79,5 com DP de 7,0) (BATSIS; VILLAREAL, 2018). E quanto ao gênero, outro fator determinante de prevalência da obesidade sarcopênica em idosos (BONNEFOY; GILBERT, 2015), também não houve diferença de ocorrência na amostra, sendo a prevalência observada em $50 \%$ em homens e mulheres.

As pesquisas sobre envelhecimento asseveram que nem todas as ILPI dispõem de assistência multimodal, embora idosos institucionalizados sejam mais propensos à falta de cuidados multidisciplinares (OLIVEIRA NETO et al., 2020).

A ILPI do estudo garante assistência integral médica e multidisciplinar. Entretanto, a mudança abrupta do estilo de vida livre para institucionalização afeta especialmente dois dos três pilares dimensionais do desempenho muscular esquelético de idosos, proposto pelo Diagrama de Tim Groheen: o fator psicossocial e de estilo de vida. Essas duas variáveis impactam diretamente na inatividade física e no consumo alimentar, em especial hipoproteico, devido à hiporexia (OLIVEIRA NETO et al., 2020; TIELAND; TROUWBORST; CLARK, 2018).

Em outros termos, existem fatores extrínsecos associados ao bem-estar biopsicossocial do idoso, os quais precisam ser gerenciados de forma integrada e contínua com os intrínsecos. No entanto, por vezes, esses cuidados não são totalmente resolutivos quando se trata da sinergia e da inevitável associação do envelhecimento patológico com o fisiológico. Deve-se, pois, elaborar estratégias que tenham como principal objetivo a promoção da melhor qualidade de vida, da beneficência e da dignidade ao idoso.

Idosos institucionalizados em precário estado nutricional podem atingir taxas de prevalência de 25 a $60 \%$ (FÁVARO-MOREIRA et al., 2016). Um planejamento de assistência multifacetada dentro das ILPI possibilita auxílio no retardo da progressão da sarcopenia e da obesidade sarcopênica. Já existem evidências que a adoção de exercícios físicos resistidos, uso de suplementos nutricionais e dietoterapia individualizada, protocolos de triagem para disfagia, atividades que estimulem a convivência social, devem fazer parte do plano de cuidados do idoso institucionalizado (OLIVEIRA et al., 2021; SMUKALLA et al., 2017; STRASSER et al., 2018).

Todavia, sabe-se que seguir esse modelo de assistência em todas ILPI no Brasil exige profissionais especializados na saúde do idoso e envolvem desafios na prática clínica e no gerenciamento administrativo, além de, por vezes, ser encarado pelos dirigentes como custos que culminam em ônus financeiros.

Existe evidente comprovação científica do uso de suplementos nutricionais orais e dietoterapia individua- lizada na efetividade da recuperação clínica e nutricional de idosos, o que traduz o uso desses artifícios como investimento ao cuidado e não como um dispêndio. Sabe-se que muitas das ILPI são mantidas à custa de benefícios sociais dos próprios idosos e de donativos, limitando, assim, a implementação da assistência ideal. Entretanto, a otimização dos recursos disponíveis e uma boa interação e comunicação entre as equipes podem repercutir em assertivos processos operacionais assistenciais, perpassando a modalidade preventiva, menos dispendiosa que a curativa em todos os âmbitos do cuidado.

\section{CONCLUSÕES}

Foi possível observar que grande parte dos idosos avaliados, e em especial as mulheres, apresentaram excesso de tecido corporal gorduroso, considerando-se todas a variáveis relacionadas à massa gordurosa corporal reveladas pela DXA. Em contrapartida, não foi possível identificar prevalência de deficit de massa magra, talvez, devido ao tamanho amostral limitado.

A assistência multidisciplinar a idosos institucionalizados é crucial na promoção da saúde e no retardo das consequências inerentes aos processo de senescência e senilidade.

A elaboração de estudos sobre essa temática é de suma importância, uma vez que o número populacional de idosos tem assumido nos últimos anos comportamento exponencial, sendo o último ciclo de vida o mais vulnerável a modificações quanto ao estado de saúde e às carências nutricionais.

\section{REFERÊNCIAS}

AL-SOFIANI, M. E. et al. Body Composition Changes in Diabetes and Aging. Journal of Diabet and its Complications, [s.I.], v. 33, n.6, p. 451-459, 2019.

BARBOSA, M. H. et al. Body mass index as discriminator of the lean mass deficit and excess body fat in institutionalized elderly people. Geriatric Nursing, New York, v. 36, n.3, p.1-5, 2015.

BATSIS, J. A.; VILLAREAL, D. T. Sarcopenic obesity in older adults: aetiology, epidemiology and treatment strategies. Nature Reviews Endocrinology, London, v. 14, n. 9, p. 513-537, 2018.

BAUMGARTNER, R. N. et al. Epidemiology of sarcopenia among the elderly in New Mexico. American Journal Epidemiology, Baltimore, v. 147, n. 8, p.755-763, Apr.1998.

BONNEFOY, M.; GILBERT, T. Composition corporelle et comorbidités chez le sujet âgé. Geriatric Psychologie and Neuropsychiatrie Vieillissement, [s.I.], v. 13, n. 1, p. 29-36, 2015.

BRASIL. Ministério da Saúde. Resolução RDC no 283 de 26 de setembro de 2005. Aprova o "Regulamento Técnico que define normas de funcionamento para as Instituições de Longa Permanência para Idosos, de caráter residencial" na forma do anexo desta Resolução. Órgão emissor: ANVISA - Agência Nacional de Vigilância Sanitária Disponível em: http://bvsms.saude.gov.br/bvs/saudelegis/anvisa/2005/ res0283_26_09_2005.html. Acesso em: 30 ago. 2021.

Rev. Ciênc. Méd. Biol., Salvador, v. 20, n. 3, p. 447-453, set./dez. 2021 
CAMARANO, A. A.; KANSO, S. R. As instituições de longa permanência para idosos no Brasil. Revista Brasileira de Estudos da População, Rio de Janeiro, v. 27, n. 1, p. 233-235, jan./jun.,2010.

CRUZ-JENTOFT, A. J. et al. Sarcopenia: European consensus on definition and diagnosis. Report of the European Working Group on Sarcopenia in Older People. Age Ageing, London, v. 39, n. 39, p. 412-423, 2010.

DENIC, A.; GLASSOCK, R. J.; RULE, A.D. Structural and functional changes with the aging kidney. Advances Chronic Kidney Disease, Philadelphia, v. 23, n. 1, p.19-28, Jan.2016.

FANTIN, F. et al. Adipokines and arterial stiffness in the elderly. Vascular Health and Risk Management, Auckland, v.16, n. 16, p.535-543, 2020.

FÁVARO-MOREIRA, N.C. et al. Risk Factors for Malnutrition in Older Adults: A Systematic Review of the Literature Based on Longitudinal Data. Advances in Nutrition, [s.I.], v. 7, n. 3, p. 507-522, 2016.

GUZIK, T. J.; COSENTINO, F. Epigenetics and Immunometabolism in Diabetes and Aging. Antioxid Redox Signal, Larchmont, v. 20, n. 3, p.257-274, 2018.

KELLY, T. L.; WILSON, K.E.; HEYMSFIELD, S.B. Dual energy X-Ray absorptiometry body composition reference values from NHANES. PloS ONE, London, v. 4, n. 9, 2009.

KURIYAN, R. Técnicas de composição corporal. Indian Journal Medical Research, New Delhi, v. 178, n. 5, p. 648-658, 2018.

LIPSCHITZ, D. A. Screening for nutritional status in the elderly. Primary Care, Philadelphia, v. 21, n. 1, p. 55-67, 1994.

LORENZO - LÓPEZ, L. L. et al. Nutritional determinants of frailty in older adults: A systematic review. Geriatrics, New York, v. 17, n.108, 2017.

LU, Y. C. et al. Dual-energy X-ray absorptiometryassessed adipose tissues in metabolically unhealthy normal weight Asians. Scientific Reports, [s.I.], v. 27, n. 1, 2019.

MIN, K. B.; MIN, J. Y. Android and gynoid fat percentages and serum lipid levels in United States adults. Clin. Endocrinol., Oxford, v.82, n.3, p.377-387, 2015.

MUNDI, M. S. et al. Current perspective for tube feeding in the elderly: from identifying malnutrition to providing of enteral nutrition. Clinical Interventions in Aging, [s.I], v.13, p.1353-1364, 2018.

NASIMI, N.; DABBAGHMANESH, M. H.; SOHRABI, Z. Nutritional status and body fat mass: Determinants of sarcopenia in community-dwelling older adults. Experimental Gerontology, [s.I.], v.15, n. 122, p. 67-73, 2019.

NERI, G. R. et al. Body fat distribution in obesity and the association with falls: A cohort study of Brazilian women aged 60 years and over. Maturitas, [s.I.], v. 139, p. 64-68, 2020.

OLIVEIRA, L. F. S. et al. Health-related quality of life of institutionalized older adults: Influence of physical, nutritional and self-perceived health status. Archives of Gerontology Geriatrics, Amsterdam, v. 92, 2021.

OLIVEIRA NETO, L. de et al. Factors associated with sarcopenia in institutionalized elderly. Journal of Public Health, [s.I.], Ago, p.1-8, 2020.

SILVA, A. L. S. C. et al. Avaliação antropométrica de idosos atendidos no Ambulatório de Nutrição do Centro de Referência em Assistência à Saúde do Idoso da Universidade Federal Fluminense, no município de Niterói-RJ. Demetra: Alimentação, Nutrição e Saúde, [s.I.], v. 10, n. 2, p. 361-374, 2015.

SILVA, R. S. et al. Condições de saúde de idosos institucionalizados: contribuições para ação interdisciplinar e promotora de saúde. Cadernos Brasileiros de Terapia Ocupacional, São Carlos, v. 27, n. 2, p. 345-356, 2019.

SILVEIRA, E. A. et al. Body fat percentage prediction in older adults: Agreement between Q8 anthropometric equations and DXA. Clinical Nutrition, Edinburgh, v. 40, p. 2091-2099, Apr. 2021.

SMUKALLA, S. M. et al. Dysphagia in the Elderly. Current Treatment Options in Gastroenterology, Philadelphia, v.15, n.3, p.382-396, 2017.

SOPEÑA, V. S. et al. Uso de indicadores antropométricos para avaliação da adiposidade corporal em idosos no sul do Brasil. Jornal da BRASPEN, [s.l.], v. 33, n. 1, p.39-42, 2018.

STRASSER, E. M. et al. Strength training increases skeletal muscle quality but not muscle mass in old institutionalized adults: a randomized, multiarm parallel and controlled intervention study. European Journal of Physical and Rehabilitation Medicine, Torino, v. 54, n.6, p.921-933, 2018.

TIELAND, M.; TROUWBORST, I.; CLARK, B. C. Skeletal muscle performance and ageing. Journal of Cachexia. Sarcopenia and Muscle, [s.l.], v. 9, n. 1, p.3-19, 2018.

VALENTINI, A. et al. Frailty and nutritional status in older people: the mini nutritional assessment as a screening tool for the identification of frail subjects. Clinical Interventions in Aging, Auckland, v.13, n. 13, 2018.

$\mathrm{XU}$, L. et al. Association between body composition and frailty in elder in patients. Clinical Interventions in Aging, Auckland, v. 4, n. 15, p. 313-320, 2020.

ZHOU, Y.; HOGLUND, P.; CLYNE, N. Comparison of DEXA and bioimpedance for body composition measurements in nondialysis patients with CKD. Journal of Renal Nutrition, Philadelphia, v. 29, n.1, p.33-38, Jan. 2019.

Submetido em: 19/11/2021

Aceito em: $22 / 11 / 2021$ 